\title{
FUNDAMENTAL POLYGONS
}

\author{
BY MAURICE HEINS
}

Communicated May 31, 1963

1. Siegel's theorem. The following elegant theorem is due to C. L. Siegel $[3 ; \mathbf{4}]$ :

TheOREM 1. Let $\Theta$ denote a group of Möbius transformations mapping $\Delta=\{|z|<1\}$ onto itself which is properly discontinuous at each point of $\Delta$. If the common hyperbolic area of the associated Poincaré polygons is finite, then each Poincaré polygon associated with $\Theta$ has a finite number of sides, none on $C=\{|z|=1\}$.

A group $\Theta$ satisfying the hypothesis of Siegel is necessarily of the first kind. The question arises whether there is a theorem of the Siegel type for $\Theta$ of the second kind. It is one of the objects of the present investigation to establish such a theorem and to draw consequences of this result taken in conjunction with Siegel's theorem. The other object is to study the relation between the parabolic transformations of an unrestricted $\Theta$ and the cusps of an associated noneuclidean convex fundamental polygon (n.e.c.f.p.). To be precise, we understand by this term a set $\Pi(C \Delta)$ satisfying the following conditions: (1) it is non-euclidean convex, (2) every point of $\Delta$ is $\Theta$-equivalent to a point of $\Pi$, (3) no two distinct points of int $I I$ are $\Theta$-equivalent, (4) $\Pi$ is closed in the sense of the topology of $\Delta$, (5) for each point of $\Delta$ there exists a neighborhood intersecting $\tau(\Pi)$ for only a finite set of $\tau \in \Theta,(6)$ (fr $\Pi) \cap \Delta$ is piecewise hyperbolic rectilinear. The Poincaré polygons are special cases of n.e.c.f.p. We shall refer to n.e.c.f.p. as fundamental polygons.

2. Reformulation of Siegel's theorem in terms of the quotient Riemann surface. We indicate how the condition of Siegel may be recast in terms independent of fundamental polygons.

If $\Theta$ is a group of the first kind, then the component containing $\Delta$ of the set of points at which $\Theta$ is properly discontinuous is $\Delta$. If $\Theta$ is of the second kind, the set of points at which $\Theta$ is properly discontinuous is connected and contains $\Delta$ properly. Let $\Omega$ denote $\Delta$ in the first case and the full set of points at which $\Theta$ is properly discontinuous in the second case. Let $\phi(z)=\{\tau(z) \mid \tau \in \Theta\}$, i.e. $\phi(z)$ is the orbit of a point $z$ with respect to $\Theta$. The image of $\Omega$ with respect to the

1 The work summarized in the present note was carried out by the author during the tenure of National Science Foundation Grant NSF G-25227. 
map $\phi: z \rightarrow \phi(z)$ may be endowed, and in fact in an essentially unique manner, with a Riemann surface structure rendering $\phi$ a conformal map of $\Omega$ onto $\phi(\Omega)$. We suppose that $\phi(\Omega)$ has been so endowed with a Riemann surface structure. For each point $p \in \phi(\Omega)$ the multiplicity of $\phi$ at the points of $\phi^{-1}(\{p\})$ has a common value $\partial(p)$; the set $\{\partial(p)>1\}$ clusters at no point of $\phi(\Omega)$. We designate by $\partial_{1}$ the restriction of $\partial$ to $\phi(\Delta)$.

Condition A. Let there be given a Riemann surface $F$ and a function $d$ on $F$ which takes positive integral values and is such that $\{d(p)>1\}$ clusters at no point of $F$. We say that the ordered pair $(F, d)$ satisfies the condition A provided that either (1) there does not exist a $C^{\prime \prime}$ conformal metric $\lambda$ of constant curvature -4 on $\{d(p)=1\}$ which has the property that

$$
\lambda_{\theta}(t)|t| 1-[d(q)]^{-1}
$$

possesses a finite limit at $q, d(q)>1, \theta$ being a uniformizer satisfying $\theta(0)=q$ and $\lambda_{\theta}(t)|d t|$ being the local representation of $\lambda$ for a deleted neighborhood of $q$ in terms of $\theta$; or (2) such a metric exists-in this case there is a maximal such metric $\mu$-and the area of $\{d(p)=1\}$ in the sense of the metric $\mu$ is finite.

With $F=\phi(\Delta)$ and $d=\partial_{1}$, we have: If $(F, d)$ satisfies the condition A, then the second alternative occurs. Actually, with $F$ and $d$ so specialized, there is always a metric $\mu$ and the $\mu$-area of $\{d(p)=1\}$, finite or infinite, is just the comon hyperbolic area of the fundamental polygons associated with $\Theta$. Thanks to this result and Siegel's theorem we are led to the following theorem concerning arbitrary $(F, d)$.

Theorem 2. $(F, d)$ satisfies the condition A if and only if $F$ is conformally equivalent to a compact Riemann surface less a finite set of points and $\{d(p)>1\}$ is finite.

Theorem 2, a geometric lemma describing the behavior of fundamental polygons in the neighborhood of a point of $C$ at which a $\Theta$ of the second kind is properly discontinuous, and the argument employed by Siegel in the proof of his theorem lead to

TheOREM 3. Let $\Theta$ be of the second kind. If $(\phi(\Omega), \partial)$ satisfies the condition A, then every associated fundamental polygon has a finite number of sides. If some associated fundamental polygon has a finite number of sides, then $(\phi(\Omega), \partial)$ satisfies the condition A.

For groups $\Theta$ of the second kind the condition $\mathrm{A}$ for $(\phi(\Omega), \partial)$ is equivalent to: either the complement of $\Omega$ with respect to the extended plane has at most two points, or else it has more than two 
and the area of each fundamental polygon associated with $\Theta$ in the sense of the maximal $C^{\prime \prime}$ conformal metric of constant curvature -4 on $\Omega$ is finite.

An obvious extension of the theorem of Siegel to fundamental polygons and Theorem 3 imply that for a given $\Theta$ either all the associated fundamental polygons have a finite number of sides (Fuchsian case) or else all associated fundamental polygons have infinitely many sides (Fuchsoid case). The terminology is not quite rigid and we are following that of Fatou [1] which designates $\Theta$ as Fuchsian when there exists an associated Poincaré polygon with a finite number of sides and otherwise as Fuchsoid. Another consequence of these theorems is that the Fuchsian $\Theta$ are precisely the finitely generated $\Theta$.

3. Parabolic elements and cusps of fundamental polygons of unrestricted $\Theta$. Given an arbitrary $(F, d)$, a pointlike isolated planar boundary element of $F$ is termed admitted (relative to $d$ ) provided that $d(p)=1$ in some deleted neighborhood of the given boundary element. We have shown elsewhere [2] essentially the following result connecting the parabolic transformations of a group $\Theta$ and the admitted (relative to $\partial_{1}$ ) pointlike isolated planar boundary elements of $\phi(\Delta)$ :

TheOREM 4. Given a maximal cyclic subgroup $A$ of $\Theta$ having a parabolic generator, let

$$
C(A)=\left\{\tau^{-1} A \tau \mid \tau \in \Theta\right\} .
$$

There exists a unique univalent map of the set of $C(A)$ onto the set of admitted (relative to $\partial_{1}$ ) pointlike isolated planar boundary elements of $\phi(\Delta)$ such that if $\zeta$ is the fixed point of the members different from the identity of $\tau^{-1} A \tau$ (for a fixed $\tau \in \Theta$ ), then the restriction of $\phi$ to an open disk $\Delta_{1}(\subset \Delta)$ the frontier of which is a circle tangent to $C$ at $\zeta$ is a conformal universal covering of an arbitrarily small deleted neighborhood of the boundary element corresponding to $C(A)$ provided that the radius of $\Delta_{1}$ is sufficiently small.

Here "arbitrarily small" is to be given the gloss "lying in a given deleted neighborhood of the boundary element in question."

Cusps. Periodic cusp cycles. By a cusp of a fundamental polygon associated with a given $\Theta$ is meant an ordered pair of distinct sides of the polygon having a common endpoint on $C$, which is such that the order with respect to the sensed frontier of the polygon of an inner point of the polygon is 1 when the frontier is so sensed that locally 
points of the first component precede and points of the second component of the cusp follow the vertex of the cusp (vertex $=$ common endpoint of sides). An ordered pair of cusps $\left(k_{1}, k_{2}\right)$ will be termed allowed provided that there exists $\tau$, not the identity, $\in \Theta$, mapping points of the second component of $k_{1}$ near the vertex of $k_{1}$ into points of the first component of $k_{2}$ near the vertex of $k_{2}$. Given a cusp $k$, there exists a unique maximal sequence of cusps $\left(k_{j}\right)_{m}^{n},-\infty \leqq m \leqq 0$ $\leqq n \leqq+\infty$, such that $k_{0}=k$ and $\left(k_{j}, k_{j+1}\right)$ is allowed for every finite integer $j, m \leqq j<n$. A sequence $\left(k_{j}\right)_{-\infty}^{+\infty}$ will be termed a periodic cusp sequence provided that there exists a positive integer $\nu$ such that $k_{j+\nu}=k_{j}$, all $j$. The vertex of each cusp of a periodic cusp sequence is the fixed point of a parabolic member of $\Theta$. By an examination of the image with respect to $\phi$ of the subset of the frontier of a fundamental polygon lying in $\Delta$ and use of Theorem 4 we are led to the following theorem relating equivalence classes of periodic cusp sequences (two such sequences are said to be equivalent provided that the cusps of each are the same) and admitted boundary elements.

Theorem 5. Given a fundamental polygon associated with a group $\Theta$, there exists a unique univalent map of the set of equivalence classes of periodic cusp sequences onto the set of admitted (relative to $\partial_{1}$ ) pointlike isolated planar boundary elements of $\phi(\Delta)$ such that the image with respect to $\phi$ of the set of points of the fundamental polygon sufficiently near the cusps of a member of an equivalence class is an arbitrarily small deleted neighborhood of the corresponding admitted boundary element of $\phi(\Delta)$. Further, for each $C(A)$ there are a member group and a periodic cusp sequence such that the fixed point of the members of the group other than the identity is the vertex of a cusp of the periodic cusp sequence.

Examples of Fuchsoid groups (of either kind) may be constructed where there are no parabolic transformations and yet some Poincaré polygon has infinitely many cusps. Unlike the case of Fuchsian groups of the first kind, it is possible for a cusp to be present in a Poincare polygon associated with a Fuchsoid group of the first kind although there is no parabolic member in the group.

The study of the admitted (relative to $\partial$ ) pointlike isolated planar boundary elements of $\phi(\Omega)$ for $\Theta$ of the second kind may be reduced to the situation of Theorem 5 thanks to the fact that for each such admitted pointlike isolated planar boundary element of $\phi(\Omega)$ there exists a deleted neighborhood having no points in common with $\phi(\Omega \cap C)$. Consequently, on considering a fundamental polygon and its reflexion in $C$, we are led to a complete correspondence between the admitted (relative to $\partial$ ) pointlike isolated planar boundary ele- 
ments of $\phi(\Omega)$ and the set of the equivalence classes of periodic cusp sequences and of the corresponding entities associated with the reflexion of the fundamental polygon with respect to $C$.

\section{BIBLIOGRAPHY}

1. P. Appell and E. Goursat, Théorie des fonctions algêbriques d'une variable et des transcendantes qui s'y attachent, Vol. 2 (Fonctions automorphes, P. Fatou), GauthierVillars, Paris, 1930.

2. M. Heins, On Fuchsoid groups that contain parabolic transformations, Contributions to function theory, Tata Institute of Fundamental Research, Bombay, 1960, pp. 203-210.

3. C. L. Siegel, Some remarks on discontinuous groups, Ann. of Math. (2) 46 (1945), 708-718.

4. ——, Ausgewählte Fragen der Funktionentheorie. II, Univ. Göttingen, Göttingen, 1954.

UNIVERSITY OF ILLINOIS 